\title{
Usage of Light-Emitting Diode Lighting and Visible Light Communication Technology for Temperature Control
}

\author{
Svetlana Grigoryeva, Alexander Baklanov, Aslima Alimkhanova
}

D. Serikbayev East Kazakhstan Technical University, Faculty of Information Technology and Intelligent Systems

A. K. Protazanov Str. 69, 070004 Ust-Kamenogorsk, Kazakhstan

e-mail: \{SGrigorieva, ABaklanov\}@ektu.kz

\begin{abstract}
Alexander Dmitriev
Novosibirsk State Technical University, Physics and Technology Faculty 20 Prospekt K. Marksa, 630073 Novosibirsk, Russia e-mail: alexander_dmitriev@ngs.ru
\end{abstract}

\section{György Györök}

Óbuda University, Alba Regia Technical Faculty

Budai út 45, H-8000 Székesfehérvár, Hungary

gyorok.gyorgy@amk.uni-obuda.hu

Abstract: A new control system via light-emitting diode (LED), lighting devices, is reviewed in this article. In order to transmit data, Visible Light Communication technology was used. Function block diagrams and schematic diagrams, of transmitting and receiving optical channels were used for the temperature control. Implementation of the developed system has been accomplished by the regulation of the onsite temperature control. Serial interface of data transmission has been applied in order to provide stable and reliable link via physical channels with the usage of LED lighting devices. According to the protocol of this interface, the transmitted data transforms into consecutive impulses in the accordance with the UART standard. The present work shows that asynchronous data transmission has been carried out via microcontrollers. In order to conduct the experiment presenting controlling possibilities via white LED lighting, a breadboard prototype with two modules transmitter and receiver - has been created. The conducted measurements have shown that optical channel of data transmission has high reliability, while the illumination level does not change. There is no flickering and a comfortable lighting regime is provided. 
Keywords: control system; data transmission; lighting devices; LED; Visible Light Communication; temperature

\section{Introduction}

Currently, modern control systems obtain information, in order to monitor and manage the physical processes from sensors and actuation devices, which are spatially remote from one another. Classical data transmission between modules of managing system is carried through wires. The necessity of the physical connection of sensors and actuation mechanisms, limits their flexibility, scalability and reliability of the working system. Switching to wireless communication systems will allow for reduction of costs on assembly and failure risks during operation, the risk which will lead to the generation of a cost effective and reliable system, as well as, provision of an opportunity of the organization of intellectual infrastructure. For example, popular management platforms such as, "Internet of Things", "SMART House", "Device to Device" and "Machine to Machine" use wireless technologies to build their infrastructure.

However, with the increase in the number of mobile users and devices connected to the Internet, problems arise in ensuring communication, data exchange and the fast acquisition of information. Most international analytical agencies give high marks to the growth in the number of connected devices in the world. In 2016 during the "Internet of Things World" conference, which was held in Santa Clara (USA), company SigFox quoted the predicted data from different companies. The values differed by the order of magnitude. For example, "Gartner" company's analytics claimed that the amount of connecting devices would reach 21 billion by 2020, while Intel company's specialists predicted the value of 200 billion devices for the same year [1]. According to the report of Cisco Visual Networking Index, the seven times increase of volume of mobile data transmission is being expected in the period since 2016 to 2021, and the amount of mobile devices per capita would reach 11.6 billion to 2021 [2]. In May 2019, Strategy Analytics company's experts published data on Internet connections of 22 billion devices [3]. Presented estimations, as well as, real data shows the necessity of the implementation of network based architectures, with high through-put, which will be able to satisfy rising exigency in the resource of wireless network.

Many types of technologies and standards have been developed, depending on the purpose and requirements for wireless systems. Technologies that transmit data in the radio frequency range have become widespread. The growth in service consumption in wireless networks has led to a shortage of radio frequency resources. To meet the growing demand, either an increase in bandwidth or an increase in spectral efficiency should be used. However, the increase in spectral efficiency is slow and cannot meet the rapidly growing demand. In parallel with 
the development of technology in the radio frequency field, there is a great potential for use in the optical range.

Additional usage of optical frequencies allows for the solution of problems related to the spectrum crunch, in wireless connections, on the basis of radio frequency. In Optical Wireless Connections (OWC) it is possible to use three main frequency bands - ultraviolet, infra-red and visible-light. Within the ambit of the last two bands, the link by means of visible-light (Visible Light Communications, VLC), wireless optics (Free Space Optics, FSO) and the link by means of optical camera (Optical Camera Communications, OCC) are possible [4] [5]. OWC technology has a variety of unique advantages such as broad spectrum, high data transmission speed, low delay, high security, immunity to radio frequent electromagnetic disturbance, available licensure, low cost and low power consumption [5-8]. It may be noted that there are publications which present comparison of OWC technologies on various aspects more carefully [9-13].

With the advent of white LEDs used for lighting, Visible Light Communication technology is rapidly developing. This technology allows a light source, in addition to lighting, to transmit information using the same light signal. Visible range of wavelength is $370-780 \mathrm{~nm}$ provides transmission capacity of $\sim 400-800$ $\mathrm{THz}$, which is ten thousand times more than in the radio frequency bandwidth [5] [6] [11]. The first demonstration of wireless data transmission through a flashing LED was demonstrated by Harald Haas, a professor at the University of Edinburgh, during the TED World Conference in Edinburgh, Scotland in 2011 [14]. Despite the fact that the technology has been developed and researched over the last 10 years, the transmitting systems with the data transmitting speed of a few $\mathrm{Gb} / \mathrm{s}$ have already been demonstrated [15-17]. Scientists claim data transfer rates from $20 \mathrm{Mbps}$ to $40 \mathrm{Gbps}$, depending on the design of the LED, which is confirmed by the research results [18].

In addition to the listed advantages of OWC technologies, it is necessary to note the attractive aspects of VLC. For the deployment (organization) of the VLC system, there is a ready-made infrastructure of transmitting devices - LED lamps. LED lighting technology supports the concept of green energy and uses energy efficient lighting sources.

In the long term, the VLC systems can be used in the wide range of applications. There are problems which limit the implementation of the VLC technology. In article [7], two main tasks are identified that need to be solved - elimination of LED flickering during data transmission and support for dimming in the lighting system. These problems are caused by the simultaneous use of luminaires directly for lighting, and also additionally for data transmission. The problems associated with the optical properties of light sources are also highlighted: high losses in the path; intersymbol interference caused by multipath; interference caused by artificial light; nonlinearity of the electro-optical response of the LED. Article [19] discusses issues related to uplink transmission and integration into the existing 
information and communication structure. The articles [13] [20] provide an overview of the main problems arising in the design of communication using VLC technology. Light source problems (flicker, blackout, line of sight and interference) and wireless communication problems (uplink and mobility) are discussed.

Today VLC technology using LED lighting is in the research and development stage. For implementation in the field of communication and wide accessibility of users, it is necessary to solve many problems. The authors have published the results of the developed systems for the transmission of audio signals [21] and symbolic data [22]. The present work suggests a new idea to use the technology of signals transmission by means of LED lighting in automated control systems by physical parameters. Previously, the authors have developed and presented [23] an automated LED lighting control system on the basis of which the implementation of VLC technology is proposed.

\section{The Construction of the Temperature Control System with the Usage of the VLC Technology}

Automated control system actuator, heater, indoors, depending on the required temperature, has been suggested and developed, on the basis of the VLC technology. Structural scheme is shown in Fig. 1. The objectives of the control system are to measure the temperature in the room, control the heating element, and maintain the set temperature in the room. Temperature sensor is located in LED device which is also information transmitter - in the given case - current temperature. Actuator of the temperature regulator and information receiver are located remotely from the signal transmitter. Constructively, the transmitter and the temperature sensor are one device, while the receiver and the actuator are another device.

Fig. 2 shows the scheme of transmitting device. The temperature sensor is used for the temperature control, which allows to transmit measured data to the microcontroller. The values of the current temperature are shown on the indicator. The signal received from the microcontroller output is added in order to transmit data by means of the VLC technology to the power supply of LED. This signal is formed in accordance with the UART technology (Universal Asynchronous Receiver-Transmitter). Since the reliable work of LED requires the usage of constant current [24], a current stabilizer, has been added to the power supply. Thus, in order for LED to transmit data stably, the switch has been used, which made it possible to connect proceeding signals, by the UART and supply the constant current for the LED. The LED in the optical channel has alternating light in accordance with the signal proceeding from the microcontroller. 


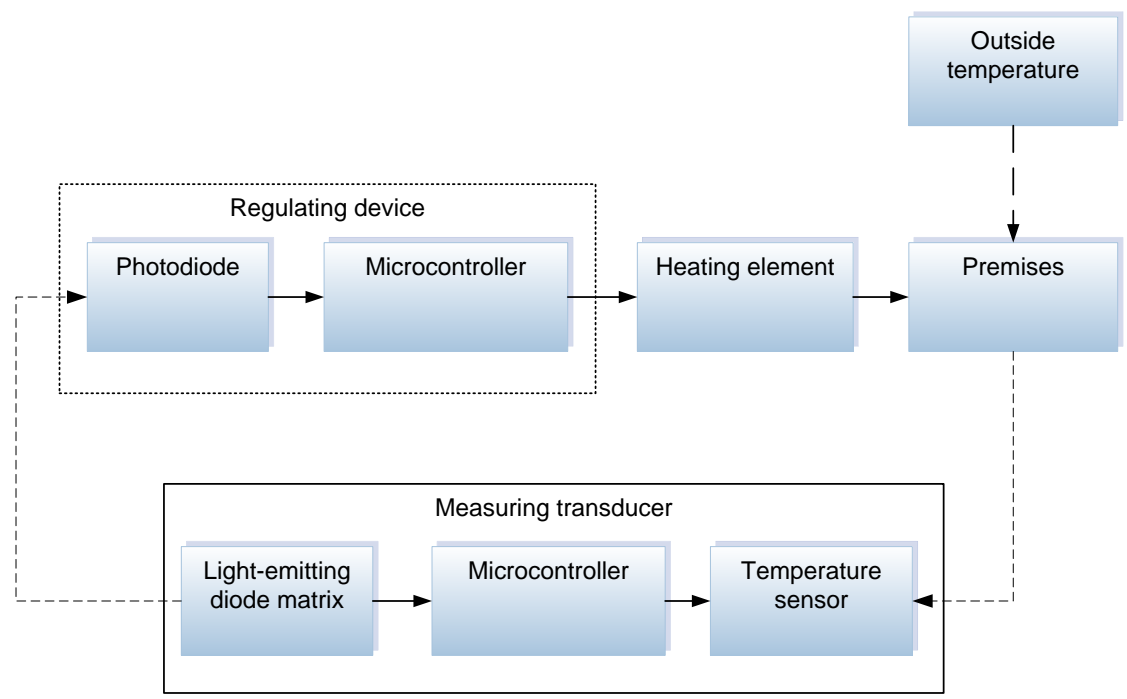

Figure 1

Structural scheme automated control system indoors temperature based on VLC technology

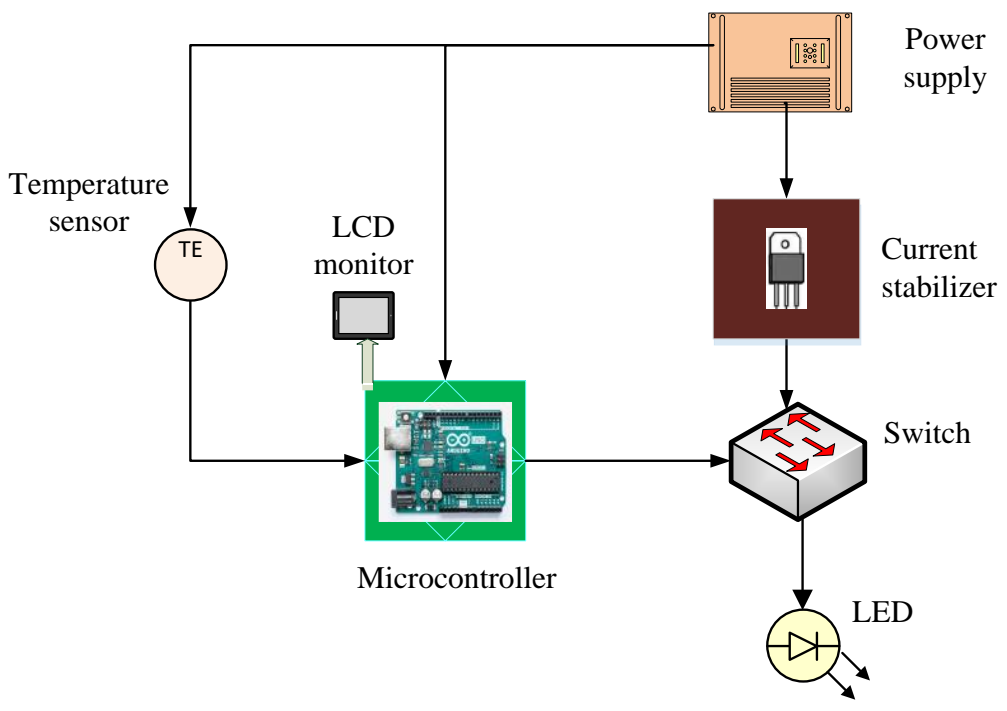

Figure 2

Transmitter function block diagram

Fig. 3 shows the scheme of the receiving device. The receiver's task is to transform light pulses received from emitter into electric signals. These signals by passing through amplifier are transferred to the shaper where they are turned into impulses of the TTL form (Transistor-Transistor Logic) for the further treatment 
by microcontroller. Microcontroller deciphers the information and processes the algorithm for the control of actuator depending on the assigned task. The received data on the current temperature is shown on the indicator.

Schematic diagrams have been developed in accordance with the function block diagrams.

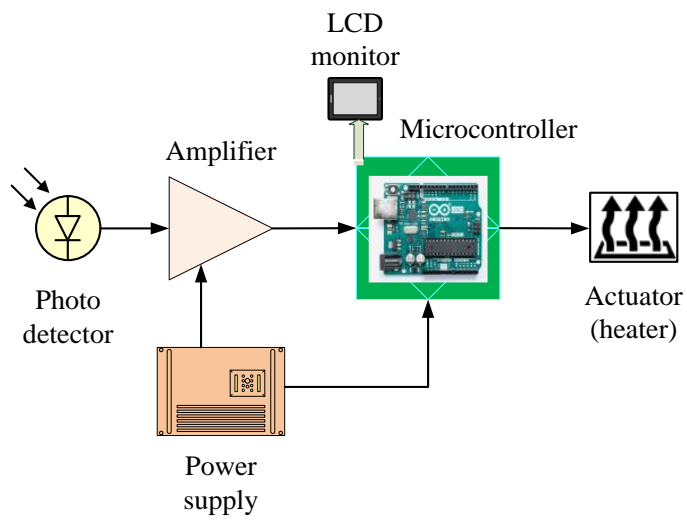

Figure 3

Receiver function block diagram

The schematic diagram of the transmitting device, is presented in Fig. 4. The transmitter's concept resides in the fact that the temperature sensor, with the transmitter, is inboarded with the LED illuminator. The transmitter sends the data of the temperature, by means of the LED of illuminator.

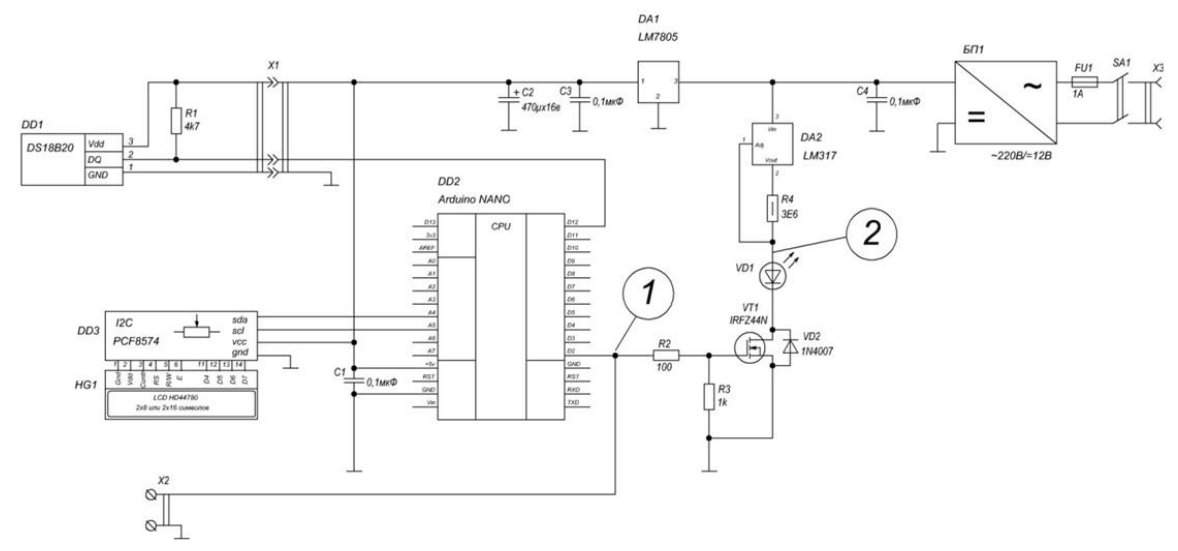

Figure 4

Transmitter circuit diagram 
The values, upon request, are transmitted from the DD1 temperature sensor to the DD2 microcontroller, where they are recorded in temporal storage registers, processed and afterwards, displayed on the HG1 indicator. The information necessary for the monitoring and control is also displayed on the HG1 indicator. The value of temperature from the temporal storage registers after transformation proceeds into the FIFO buffer of the transmitter-receiver UART of the microcontroller, where, by the means of consequent asynchronous data transmission, bits proceed to the VT1 transistor gate through the TX microcontroller output and R2 resistor. A transistor switches, from allowing, to forbidding, the current flow through LED VD1, by working in key mode according to the potential on the gate.

The DS18B20 integral temperature sensor has been used as the DD1 temperature sensor. The required drivers and connecting programs for this sensor are publicly available. The R1 resistor with the nominal value of $4.7 \mathrm{k} \Omega$ is recommended by the manufacturer of DS18B20 microchip.

ATmega328P microchip, which is a part of the Arduino NANO and Arduino Uno boards, has been chosen as the DD2 microcontroller.

The HG1 indicator is liquid-crystal indicator LCD1602 with the $\mathrm{I}^{2} \mathrm{C}$ module.

IRFZ44N has been chosen as the VT1 transistor, but it is possible to use any nchannel MOSFET designed for the usage of light emitting diode VD1. The VD2 diode is necessary in order to shunt the transistor from current impulse, which occurs due to power-cut. This diode is located inside the IRFZ44N transistor case.

The R2 resistor in the gate circuit limits the current at transistor opening. The R3 resistor is connected to the gate in order to have reliable and fast transistor closure. It eliminates the possibility of the occurrence of the unconfigured state of the transistor and as a consequence will eliminate the presence of random errors in transmitter which might have occurred for that reason.

The VD1 - white LED powerful current of $700 \mathrm{~mA}$ which has possibility of installation on the radiator cooling system.

The DA2 microchip - integrated stabilizer LM317 is included in the current stabilization circuit. The R4 resistor is a shunt for the stabilizer. In our case, the $\mathrm{R} 4$ 's resistance is $3.6 \Omega$.

The DA1 microchip - an integral stabilizer LM7805 of fixed voltage of $5 \mathrm{~V}$ provides power supply for microprocessor's board, indicator and temperature sensor.

The power source PS1provides power supply from alternating current system of $\sim 230 \mathrm{~V}, 50 \mathrm{~Hz}$. It is impulse and produces the constant regulated voltage of $12 \mathrm{~V}$ and the current of $1.5 \mathrm{~A}$.

$\mathrm{C} 1, \mathrm{C} 3, \mathrm{C} 4$ are filters capacitors, and $\mathrm{C} 2$ is a bulk capacitor. 
Schematic diagram of the receiving device is presented in the Fig. 5.

The received light signal is transformed by the VD1 photodiode into photo current and through the DA1 amplifier-convertor of the current into voltage and through the C2 separating capacitor it proceeds to the DA2 amplifier's input. Afterwards it proceeds from the DA2 amplifier's output through the C3-R6 differentiating circuit to the DD1 logical gate's input, which performs the duty of buffering element and simultaneously inverts the signal for the further processing. Inverting the signal is necessary in order to bring the waveform of the required polarity to the UART transmitter-receiver, since photodiode connected with the reverse polarity generates inverse current impulses. The signal proceeds from the inverter's output to the UART transmitter-receiver input of DD2 microcontroller, where it is decrypted. The received data is recorded into the registers of temporary variables storage, where it afterwards proceeds for further processing and displaying onto the liquid-crystal sensor. The SB1-SB3 buttons are used for the changes of temperature regulation. Further data processing depends on which data is being transmitted and what it is used for. In our system, we transmit the value of the temperature to regulator - heating element EK1.

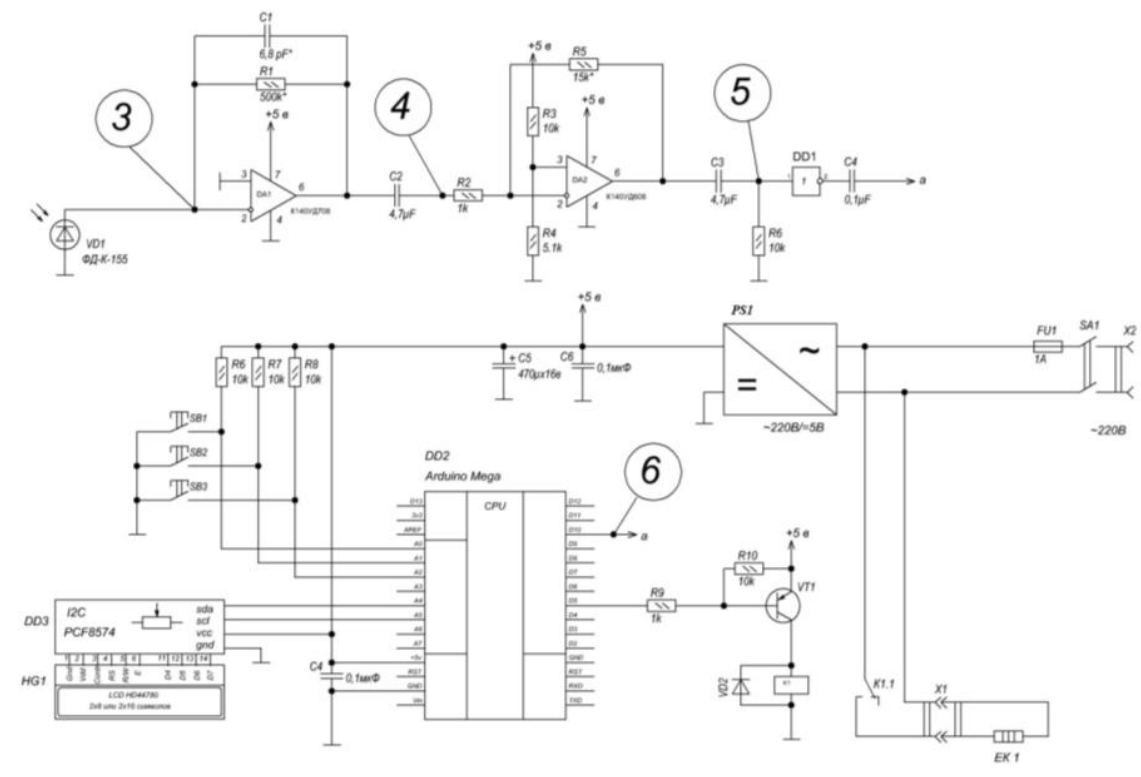

Figure 5

Receiver circuit diagram

The FD-K-155 photodiode has been chosen as the VD1 photodiode. The DA1 microchip has been developed on the basis of the KR140UD708 operational amplifier, while the DA2 has been developed on basis of the KR140UD608 operational amplifier. 
The $\mathrm{C} 1$ condenser connected in parallel to the $\mathrm{R} 1$ resistor of negative circuit reentrancy is necessary in order to reduce the gain of high-frequency noise. Its nominal has to be matched specifically carefully. The R1, R2 and R5 resisters affecting the gain coefficient of operational amplifiers, as well as, the $\mathrm{C} 2$ and $\mathrm{C} 3$ condensers, which define the steepness of pulse front and droop, have been matched so that at the rate of 9600 baud, the form of the signal in the control points 4, 5 and 6 is approximated the most closely to the TTL impulses.

It is necessary to control a backhaul and a signal format whilst adjusting the receiving part of the device. Points for the recording of oscillograms, which are necessary for the research and calibration of the whole system in data transmission mode have been marked 1-6 in the schematic diagram.

\section{The Experimental Results Settings of the Receiver}

We have carried out research in order to optimize the system's values. Oscillograph has been controlling the presence and the form of the signal during the data transmission. The level and the form of the output signal from transmitter's microcontroller and the signal transmitted onto the receiver's microcontroller are crucial. The most crucial points of control are marked on the schematic diagrams of the transmitter and the receiver as markers 1-6. The signal proceeding from the transmitter's microcontroller is a pattern of the form and amount of impulses (marker 1). Oscillogram at this point is controlled by the second beam of oscillograph and is present at all oscillograms of control points for synchronization. All other oscillograms of control points are marked by the first beam of the oscillograph.

The signal to LED (marker 2) proceeds from transistor, which is the switch of the transmitting part. The control of this point provides the information on the switch working capacity. In case of the failure it is no longer able to be opened or closed or it happens incompletely. The form of the signal is shown in Fig. 6.

It is crucial to control the signal's level after all electrical components until entering the microcontroller during the work of the receiving device. The control of the signal after photodiode (marker 3 ) shows the working capacity of the photo current source - in our case the FD-K-155 photodiode of the receiving part of the apparatus. The oscillogram (Fig. 7) shows that the amplitude of the signal on the photodiode is very low $(0.8 \mathrm{~V})$, given the oscillogram was taken when the LED of the transmitter and photodiode were almost immediately adjacent. Along with the increasing of the distance between LED and photodiode the amplitude of the signal was decreasing significantly. Therefore, for the further work with the signal of such amplitude the amplifier is required. 


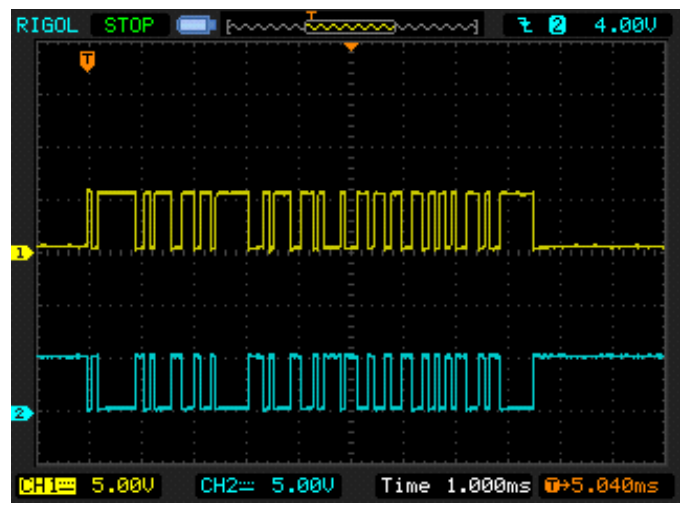

Figure 6

Waveform of the signal from the transmitter

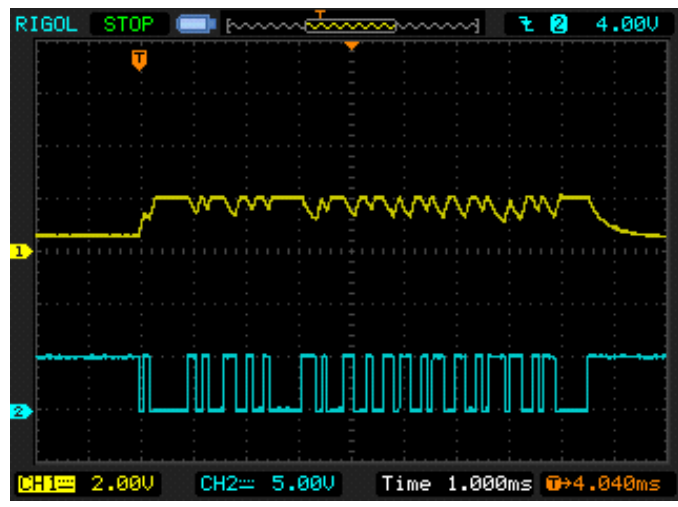

Figure 7

Waveform of the signal on the photodiode of the receiver

The measurement of the signal for the work adjustments of the first cascade of photo current amplification is included. The form of the signal at this point (marker 4) can be seen in Fig. 8. According to the form of the signal at this point, you can adjust the gain coefficient of the first stage on the K561LN2 microchip, by selecting a feedback resistor. The diagram has a condenser shunted to the ground and is meant for the elimination or the minimizing of the high-frequency noise, at the output of the first cascade of amplification.

According to the oscillogram's signal after the second cascade of amplification (marker 5) the coefficient of amplification at the second K561LN2 microchip is adjusted by the resistor selection. The form of the signal is shown in Fig. 9. 


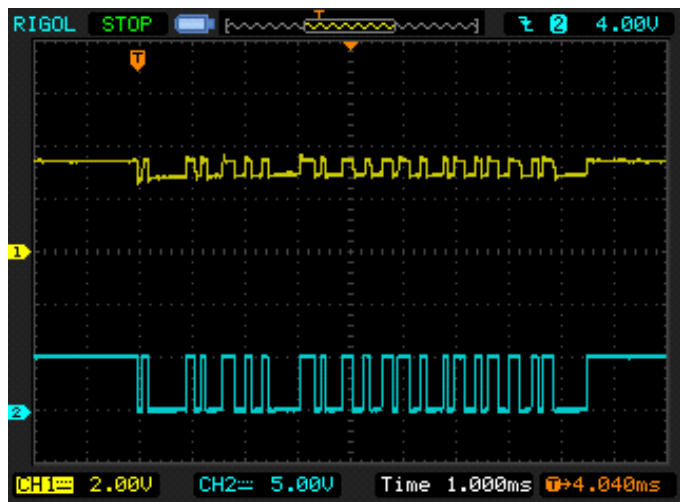

Figure 8

Waveform of the signal after the first cascade of receiver's amplification

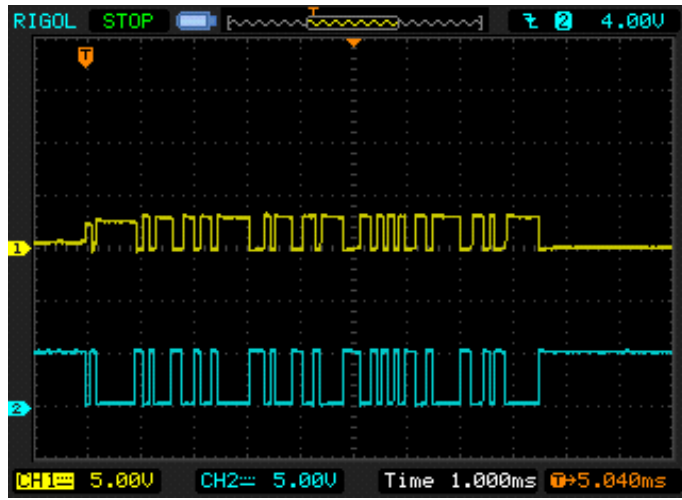

Figure 9

Waveform of the signal after the second cascade of the receiver's amplification

After the buffer amplifier-inverter (marker 6), the signal that almost completely coincides with the etalon, can be seen. The form of the signal at this point is shown in Fig. 10.

Adjustment of all parts of the receiver's scheme has been conducted with the synchronization of the signal through the second channel of the oscillograph (etalon one) in order to see each impulse separately during transmission. Adjusting cascades of the amplifier at each control point, we have achieved similar form of the signal and impulse length which provides stable work of the entire system. Identity of the transmitted and received signals ensures reliability and accuracy of the system's performance. 


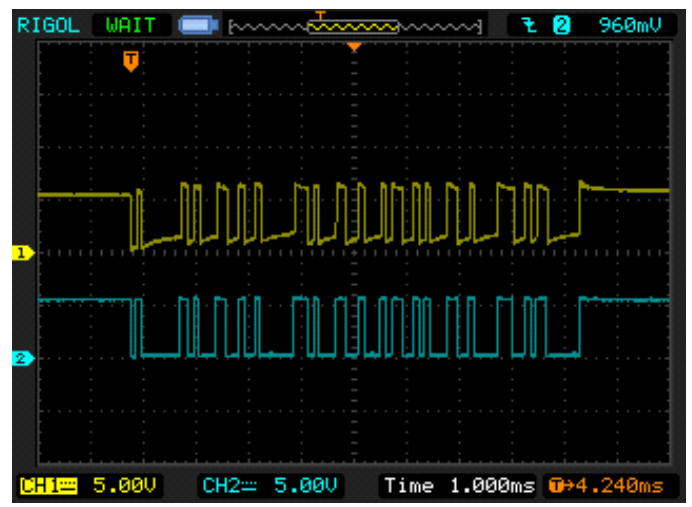

Figure 10

Waveform of the signal after the buffer amplifier-inverter of the receiver

\section{Test Environment of Automated Control System of Temperature}

Fig. 11 shows structural diagram of the test installation, of automated control system of a heater, in accordance with VLC technology, which has been developed on the basis of function blocks and schematic diagrams.

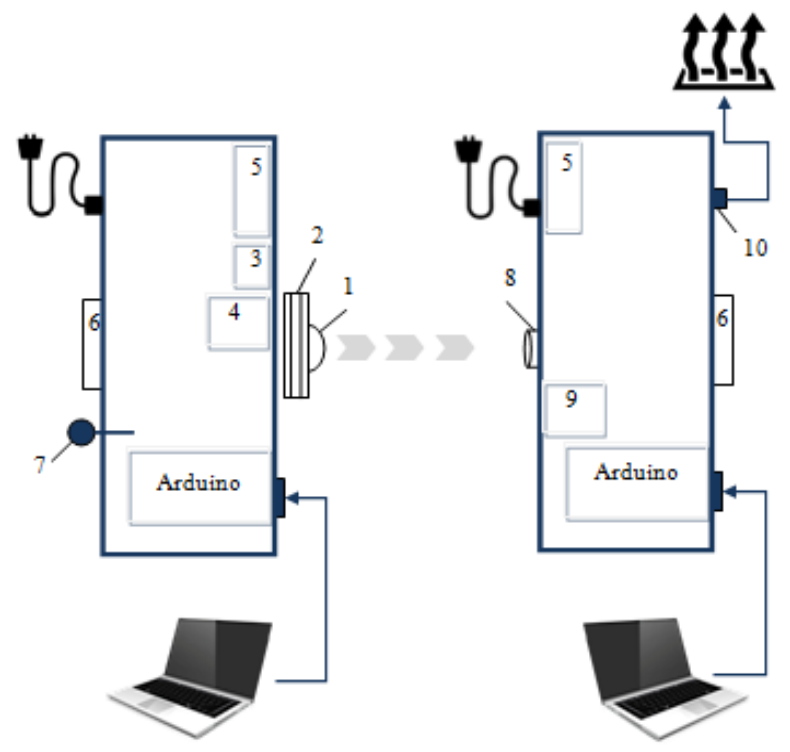

Figure 11

Structural diagram of test installation 
Power of LED matrix (1) is $3 \mathrm{~W}$ and constant current of $700 \mathrm{~mA}$ is attached to the aluminum radiator (2). The radiator is installed with the gap on the plate of getinaks. The radiator has been designed and manufactured, so that there wouldn't be any overheating of the crystal's light-emitting array, due to continuous performance [25]. The gap is necessary for providing heat elimination, from the radiator. On the opposite side of the front panel, where the light emitting diode is placed, the current stabilizer (3), the commutator (4), the power source (5) and Arduino Nano board are suited. The sketch loading is provided through mini USB-port of the microcontroller.

The DS18B20 integral digital sensor of temperature manufactured by the Dallas Semiconductor company in the TO-92 (7) case, as well as, the LCD1602 with the $\mathrm{I}^{2} \mathrm{C}$ (6) module for the monitoring of the control system values are attached to the front side of the back panel. Fig. 12 shows physical configuration of the transmitting device.
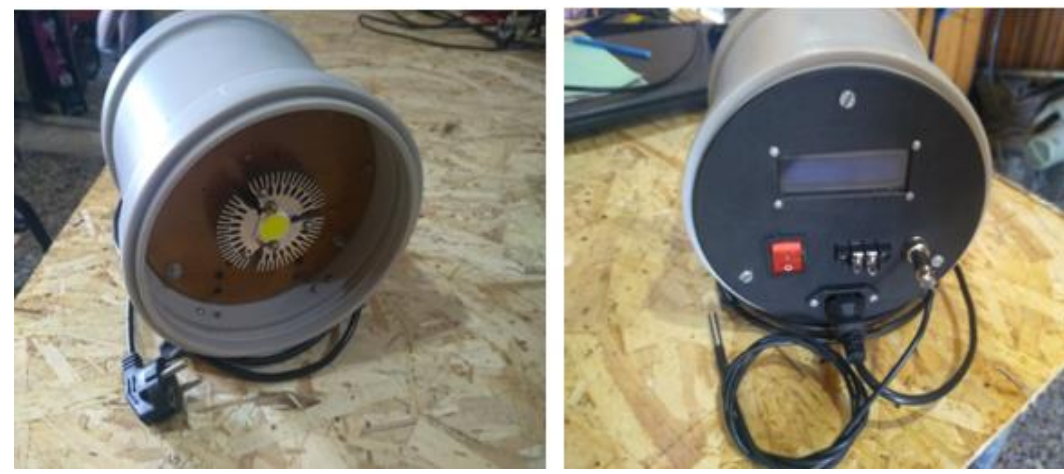

a) view of the working panel, b) view of the back panel

Figure 12

Physical configuration of the transmitting device

The FD-K-155 photodiode (8), which is attached to the external panel, is used for the receiving of LED impulses. The amplifier developed on the basis of the K561LN2 microchip (9), power source (5) and Arduino Nano microcontroller are suited onto the interior side. In order to control the signal of the exterior back panel the LCD monitor (7), the output for heating source control (10), the buttons for the assignment of required values of temperature have been placed. Fig. 13 shows physical configuration of the receiving device.

Modulating methods used for radio-frequency communication [26-28] can be used in the VLC systems, as well as, specific modulating methods such as laser femtosecond systems [29] can be implemented. 

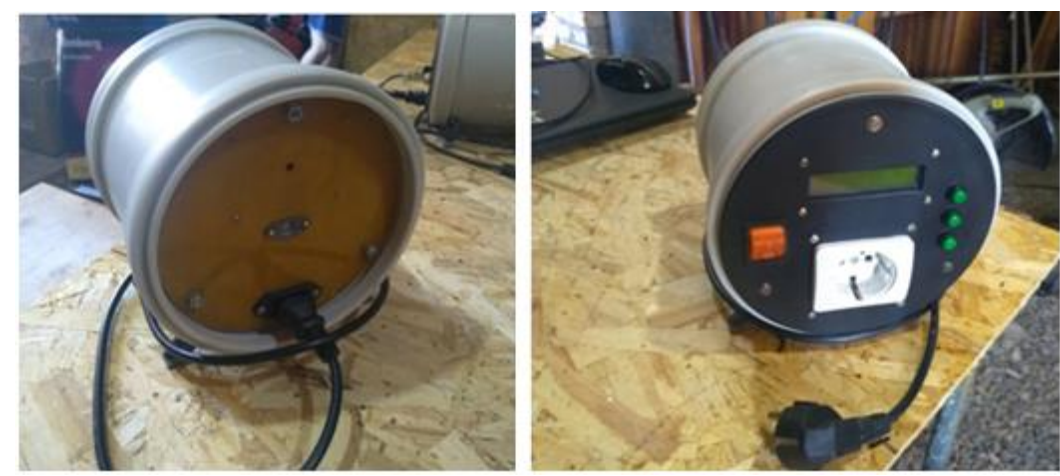

a) view of the working panel, b) view of the back panel

Figure 13

Physical configuration of the receiving device

In order to organize the system of the temperature monitoring and heater control through optical wireless connection with LED lighting element, the UART (Universal Asynchronous Receiver-Transmitter) technology has been used. In our case in accordance with the present technology the microcontroller has been programmed.

During the data transmission the converting of the transmitted data into coherent manner is carried out in the way so that it is possible to transfer it through the digital line to another similar device.

The data transmission in the UART is carried out by one bit at a time in equal time length. This time length is defined by target velocity of the UART. The choice of the UART speed depends on the physical capabilities (parameters) of the electronic components of the receive/transmit path. Data transfer speed was not the main criterion. The task was to obtain reliable data on the ambient temperature, as well as to maintain the required level of illumination and the absence of LED flickering. For our particular connection, the baud rate is set to 9600 baud. This velocity has been chosen experimentally as the most optimal, for the systems electrical elements, for transmitting and receiving devices.

The control system uses two devices for data transmission - a receiver and a transmitter. Since each device is equipped with a microcontroller, two separate projects were developed.

Projects of programs for Arduino Nano microcontrollers were developed in the visual programming environment FLProg [30] using the graphical programming language FBD (Function Block Diagram).

When programming the receiver according to the functional and logical principle, five blocks were allocated: receiving data from the UART port, separating the 
integer and fractional parts of the temperature value, presentation of data on the LCD display, setting the temperature in manual mode, program for the controller.

To operate the transmitter, three block diagrams were written: a program for polling a temperature sensor, a program for operating the UART port, a program for presenting data on the LCD display.

Here, we describe the algorithm for the operation of the temperature control system, using the developed experimental stand.

1) Turn on the receiver and transmitter. The receiving device must be located opposite the transmitting device. In our case, the maximum value of the distance between the receiver and the transmitter is $0.80-0.85 \mathrm{~m}$.

2) The user sets the preset temperature using the buttons on the receiver. The set temperature is displayed on the monitor.

3) The temperature sensor located on the transmitting device determines the value of the ambient temperature and transmits it to the microcontroller.

4) The microcontroller processes the data, displays the temperature value on the monitor and transmits it through the LED.

5) A photodiode located on the receiving device reads the signal and transmits it through an amplifier to the microcontroller.

6) The microcontroller processes the signal and displays the received temperature data on the monitor. If the obtained temperature value is less than the set value, then the heating element turns on and the monitor displays "heat on". If the value of the measured temperature is higher than the set one, then the heating element is not switched on / off and the monitor displays "heat off". The range of regulation of the set temperature value is set by software.

The suggested control system is useful for the regulation of other physical processes, which at the present, rely on the wire connection systems or wireless systems with the radio wave usage. The transition to wireless control of technological processes is especially crucial. For example, the work [31], considers the usage of LED lighting in agricultural field for optimal provision or substitution of natural light for the plant production. Present artificial lighting infrastructure can become the platform for the organization of intellectual control systems of value, without a large financial investment.

\section{Conclusions}

As a result of the conducted experiments, stable data transmission, by means of white LED lighting and the coding by means of UART technology, has been achieved. The obtained results have allowed to provide for the regulation of temperature indoors, by means of optical wireless communication, via a LED lighting system. The base of the developed system, are inexpensive ATmega328P 
microcontrollers. Such an approach makes wireless systems, which are based on the usage of LED devices, a good prospect. This being said, the main features of the suggested system, is the simplicity of construction and low production cost of the radio-electronic elements. The suggested control system provides fast transmission of the data needed for the current temperature and regulation.

The advantages of the manufacture of control systems, with VLC technology is the organization of the values regulation system, providing the work of a "Smart House" and the intellectual energetic systems. The implementation of such developments is stimulated by the exponential growth in the use of LED lighting fixtures.

\section{References}

[1] World experience and development prospects of the industrial Internet of things in Russia. Link: https://www.crn.ru/news

[2] Cisco Visual Networking Index: Global Mobile Data Traffic Forecast Update, 2016-2021, White Paper, 2017, p. 35

[3] Internet of Things, IoT, M2M world market. Link: http://tadviser.com/index.php

[4] Visible light for broadband communications. Report ITU-R SM.2422-1. Geneva, 2019, Link: https://www.itu.int/pub/R-REP-SM.2422

[5] H. Haas, J. Elmirghani, I. White: Optical wireless communication, Royal Society, Vol. 378, Issue 2169, 2020, doi.org/10.1098/rsta.2020.0051

[6] S. R. Teli1, S. Zvanovec, Z. Ghassemlooy: Optical Internet of Things within 5G: Applications and Challenges, IEEE International Conference on Internet of Things and Intelligence System, 2018, DOI: 10.1109/IOTAIS.2018.8600894

[7] I. Alimi, A. Shahpari, A. Sousa, R. Ferreira, P. Monteiro and A. Teixeira: Challenges and Opportunities of Optical Wireless Communication Technologies, 2017, DOI: 10.5772/intechopen.69113

[8] Z. Ghassemlooy, S. Amon, M. Uysal, Z. Xu, J. Cheng: Emerging optical wireless communications advances and challenges, IEEE Journal on Selected Areas in Communications, Vol. 33 (9), 2015, pp. 1738-1749

[9] M. Chowdhury, M. Hossan, A. Islam, Y. Jang: A Comparative Survey of Optical Wireless Technologies: Architectures and Applications, IEEE Access, Vol. 6, 2018, pp. 9819-9840, DOI: 10.1109/ACCESS.2018.2792419

[10] A. Sevincer, A. Bhattarai, M. Bilgi, M. Yuksel, N. Pala: LIGHTNETs: smart LIGHTing and mobile optical wireless networks - a survey, IEEE Communication Surveys \& Tutorials, Vol. 15, No. 4, 2013, pp. 1620-1641, DOI: $10.1109 /$ SURV.2013.032713.00150 
[11] D. Karunatilaka, F. Zafar, V. Kalavally, R. Parthiban: LED based indoor visible light communication: State of the art, IEEE Communication Surveys \& Tutorials, Vol. 17, No. 3, 2015, pp. 1649-1678

[12] M. Chowdhury, Md. Shahjalal, M. Hasan, Y. Jang: The Role of Optical Wireless Communication Technologies in 5G/6G and IoT Solutions: Prospects, Directions, and Challenges, Applied Sciences, 2019, DOI: 10.3390/app9204367

[13] O. Alsulami, A. T. Hussein, M. T. Alresheedi and J. M. H. Elmirghani: Optical Wireless Communication Systems, A Survey, 2018, DOI: 10.13140/RG.2.2.11751.09129

[14] H. Haas: Wireless data from every light bulb, TED Global, Edinburgh, July 2011

[15] A. M. Khalid, G. Cossu, R. Corsini, P. Choudhury, E. Ciaramella: 1-Gb/s transmission over a phosphorescent white LED by using rate adaptive discrete multitone modulation, IEEE Photon. J., Vol. 4, Issue 5, 2012, pp. $1465-1473$

[16] A. Azhar, T. Tran, D. O'Brien: A Gigabit/s indoor wireless transmission using MIMO-OFDM visible-light communications, IEEE Photon. Technol. Lett., Vol. 25, No. 2, 2013, pp. 171-174

[17] R. X. G. Ferreira, E. Xie, J. J. D. McKendry, et al.: High bandwidth GaNbased micro-LEDs for visible light communication, IEEE Photonics Technology Letters, Vol. 28 (19), 2016, DOI:10.1109/LPT.2016.2581318

[18] D. O'Brien, S. Rajbhandari, H. Chun: Transmitter and receiver technologies for optical wireless, Royal Society, Vol. 378, Issue 2169, 2020, doi.org/10.1098/rsta.2019.0182

[19] S. U. Rehman; S. Ullah, P. Chong, S. Yongchareon: Visible Light Communication: A System Perspective-Overview and Challenges, Sensors, Vol. 19 (5), N 1153, 2019, DOI:10.3390/s19051153

[20] L. Matheus, A. Vieira; F. M. Luiz, M. Vieira, Omprakash Gnawali: Visible Light Communication: Concepts, Applications and Challenges, IEEE Communications Surveys \& Tutorials, Vol. 21, Issue 4, 2019, pp. 32043237, DOI:10.1109/COMST.2019.2913348

[21] A. Baklanov, S. Grigoryeva, A. Alimkhanova, E. Grigoryev, V. Sayun: Audio Transmission System Using White LEDs, International Siberian Conference on Control and Communications (SIBCON), Tomsk, Russia, 2019, DOI:10.1109/SIBCON.2019.8729564

[22] E. A. Grigoryev, A. E. Baklanov, S. V. Grigoryeva, A. Zh. Alimkhanova, V. M. Sayun: A New Approach to Physical Encoding in VLC Data Transmission Technology, $21^{\text {st }}$ International Conference on 
Micro/Nanotechnologies and Electron Devices (EDM), Erlagol, Russia, 2020

[23] S. Grigoryeva, A. Baklanov, V. Sayun, D. Titov, Ye. Grigoryev: Analysis energy efficiency of automated control system of LED lighting, International Siberian Conference on Control and Communications (SIBCON), 2017, DOI:10.1109/SIBCON.2017.7998488

[24] A. Baklanov, S. Grigoryeva, Gy. Györök: Control of LED Lighting Equipment with Robustness Elements, Acta Polytechnica Hungarica, Vol. 13, No. 5, 2016, pp. 105-119, DOI:10.12700/APH.13.5.2016.5.6

[25] Y. A. Grigoryev, V. M. Sayun, S. V. Grigoryeva, D. N. Titov: Study of Illumination Properties of High-Power LEDs in Various Temperature Conditions, $18^{\text {th }}$ International Conference of Young Specialists on Micro/Nanotechnologies and Electron Devices (EDM), Erlagol, Russia, 2017, pp. 309-313, DOI:10.1109/EDM.2017.7981762

[26] F. A. Dahri, S. Ali, M. M. Jawaid: A Review of Modulation Schemes for Visible Light Communication, International Journal of Computer Science and Network Security, Vol. 18, No. 2, 2018, pp. 117-122

[27] A. Aliaberi, P. C. Sofotasios, S. Muhaidat: Modulation Schemes for Visible Light Communications, Advanced Communication Technologies and Networking (CommNet), 2019, DOI:10.1109/COMMNET.2019.8742376

[28] C. Manimegalai, S. Gauni, N. Raghavan, T. Rao: Investigations on suitable modulation techniques for visible light communications, International Conference on Wireless Communications, Signal Processing and Networking (WiSPNET), 2017, pp. 1818-1822, DOI:10.1109/WiSPNET.2017.8300075

[29] E. V. Baklanov, N. N. Golovin, A. K. Dmitriev, S. Grigor'eva: A femtosecond frequency standard with an external high-finesse interferometer, Optics and Spectroscopy, Vol. 121, No. 6, 2016, pp. 930933, DOI:10.1134/S0030400X16120055

[30] FLProg. Link: https://flprog.ru/en/

[31] C. Kárász, J. Kopják: Comparative Study on Plant Type Specific LED Light Source Design Parameters, Acta Polytechnica Hungarica, Vol. 17, No. 3, 2020, pp. 71-90, DOI:10.12700/APH.17.3.2020.3.4 